\title{
Advantages and Challenges of Tailored Regimens for Drug-Resistant Tuberculosis: A StopTB Italia Look into the Future
}

This article was published in the following Dove Press journal:

Infection and Drug Resistance

\author{
Niccolò Riccardi $\mathbb{D}^{1,2}$, \\ Simone Villa $\mathbb{D}^{1,3,}$ \\ Riccardo Alagna ${ }^{1,4}$ \\ Andrea Giacomelli (1D) ${ }^{1,5}$ \\ Laura Saderi $\mathbb{D}^{1,6}$ \\ Daniela Maria Cirillo',4 \\ Giorgio Besozzi (D) \\ Giovanni Sotgiu (1D) \\ Luigi Codecasa ${ }^{1,7}$
}

\begin{abstract}
'StopTB Italia Onlus, Milan 20159, Italy; ${ }^{2}$ Department of Infectious - Tropical Diseases and Microbiology, IRCCS Sacro Cuore Don Calabria Hospital, Negrar di Valpolicella, Verona 37024, Italy; ${ }^{3}$ Centre for Multidisciplinary Research in Health Science, University of Milan, Milan 20I22, Italy; ${ }^{4}$ IRCCS San Raffaele Scientific Institute, Milan 20I32, Italy; ${ }^{5}$ III Infectious Diseases Unit, ASST Fatebenefratelli Sacco, Milan 20157, Italy; ${ }^{6}$ Clinical Epidemiology and Medical Statistics Unit, Department of Medical, Surgical and Experimental Sciences, University of Sassari, Sassari 07100, Italy; ${ }^{7}$ Regional TB Reference Centre, Istituto Villa Marelli, Niguarda Hospital, Milan 20159, Italy

*These authors contributed equally to this work
\end{abstract}

Correspondence: Niccolò Riccardi Department of Infectious - Tropical Diseases and Microbiology, IRCCS Sacro Cuore Don Calabria Hospital, Via Don A. Sempreboni N.5, 37024, Negrar di Valpolicella, Verona, Italy

Tel +39045 60l 6420

Email niccolo.riccardi@yahoo.it

\begin{abstract}
The emerge of drug-resistant tuberculosis (TB) strain in recent decades is hampering the efforts of the international community to eliminate the disease worldwide. The World Health Organization (WHO) has drafted many strategies to achieve this ambitious goal. In the very beginning, the aim was to standardize inadequate regimens used in many countries and, thereafter, evolved to tackle the social determinants which hinder TB elimination. However, following the path of narrowing the clinical vision to deal with TB, there is an increased need to personalize the treatment considering both patients and pathogen unique characteristics. In our narrative review, we report the advantages and the backwards in developing a method to implement the concept of precision medicine to the treatment of TB. In this dissertation, we highlight the importance to address different aspects of the diseases encompassing the host and pathogen features, as well as the needs to further implement an adequate follow-up based on the available resources. Nevertheless, many things may hamper the vision of precision medicine in TB, such as the complexity and the costs to develop novel compounds and the costs related to global-scale implementation of patient-centered follow-up. To achieve the ambitious goal of TB elimination, a radical change in TB treatment is needed in order to give a more comprehensive approach based both on patients' peculiarities and driven by drug susceptibility tests and whole-genome sequencing.
\end{abstract}

Keywords: tuberculosis, drug-resistance, treatment, tailored, StopTB

Tuberculosis (TB) is the leading cause of death from a single microorganism. TB therapy is the outcome of several clinical studies. ${ }^{1,2}$ After the first therapeutic trials in the 40s' and 50s' aimed at evaluating single drugs which could reduce mortality rates and increase cure rates, it was clear that more than a single drug was needed to prevent the emergence of drug-resistant TB (DR-TB) strains. The next therapeutic challenge was to combine this goal with the reduction of the long treatment duration (ie, 12-24 months), which hindered patients' adherence. The introduction of rifampicin in the 70s' helped develop an effective short-course TB therapy for drug-susceptible (DS) forms. ${ }^{1}$ A standardized regimen for DS-TB is characterized by a 2-month intensive (bactericidal) phase with four drugs (isoniazid $(\mathrm{H})$, rifampicin (R), ethambutol (E), and pyrazinamide (Z); 2HRZE) and a 4-month continuation (sterilizing) phase with HR.

DR-TB forms can be classified in the following groups: (i) isoniazid-resistant TB (Hr-TB); (ii) rifampicin-resistant TB (RR-TB); multidrug-resistant TB (MDR-TB), 
with mycobacterial strains resistant to at least isoniazid and rifampicin; pre-extensively-drug-resistant TB (pre-XDRTB) with MDR mycobacterial strains resistant to fluoroquinolones or injectable aminoglycosides; and XDR-TB with MDR mycobacterial strains resistant to both fluoroquinolones and aminoglycosides. ${ }^{2}$

New diagnostic tools that can rapidly detect DR profiles (ie, drug susceptibility tests [DSTs]) and TB drug research and development $(\mathrm{R} \& \mathrm{D})$ could be key to tackle DR-TB and prevent further transmission of difficult-totreat TB forms.

TB incident cases are 10 million annually worldwide, with 500,000 patients with DR-TB forms. ${ }^{1}$ Treatment success for RR/MDR-TB forms (75\%) is suboptimal and lower if compared with that achieved in DS forms $(85 \%)^{2,3}$ Furthermore, a quarter of the world population is estimated to be infected by Mycobacterium tuberculosis (M.tb) and 11\% (6 million people) is estimated to be infected with Hr-TB strains.

Interventions aimed at identifying at-risk subjects with latent infection and administering a preventative therapy can significantly change TB epidemiology., ${ }^{4,5}$

Tailoring regimens are key for DR-TB forms, preventing drug-drug interactions (DDIs) and drug-induced adverse events (AEs), which may reduce treatment success and completion rates. During the current decade, three new molecules were licensed for DR-TB: Bedaquiline (in 2012) (Bdq), Delamanid (in 2014) (Dlm), and Pretomanid (in 2019) (PA-824 or Pa). ${ }^{6,7}$ WHO has recommended the prescription of Bdq beyond 6 months of treatment, as well as during pregnancy, and in association with Dlm. Oral regimens should be prescribed when feasible (eg, Bdq, Pa and $\mathrm{D} \operatorname{lm})^{8}$

Ideally, successful treatment outcome can be improved prescribing effective drugs whose choice depends on DST and host biomarkers. ${ }^{6,9}$

\section{Advantages of Precision TB Treatment \\ Tailoring Regimens: Achieving Individualized Cure While Minimizing Toxicity}

In order to eliminate $\mathrm{TB}$, treatment success needs to be ensured avoiding relapse in active TB and reactivation of LTBI.

From a patient point of view, to achieve this goal, TB treatment requires to be easily administrated to patients, by ensuring safety and tolerability when prescribed with other drugs, and as short as possible ${ }^{10,11}$ to enhance treatment compliance. Developing and implementing the use of predictive host-biomarkers - which provide information on the outcome based on a therapeutic intervention - could help improve treatment outcome rates.

Firstly, at-risk behaviors like unhealthy diet (eg fatty and fried foods may exacerbate drug-related liver toxicity), malnutrition, alcohol consumption, tobacco smoking, and substance abuse need to be investigated to prevent toxicity. $^{12,13}$ Likewise, the clinician ought to explore the presence of any other co-morbidity (eg chronic obstructive pulmonary disease, HIV, diabetes mellitus) and possible ongoing treatment (eg anti-retroviral drugs, psychiatric medications, other concomitant antibiotic treatment). More importantly, because the initial treatment is always empirical clinicians should ask about a previous TB drug exposure to reduce the probabilities of unfavorable outcomes.

Ideally, clinicians would prefer to monitor anti-TB treatment by studying the pharmacokinetics/pharmacodynamics of the drugs in blood samples to adequately adjust the dosage $\mathrm{e}^{12,13}$ to maximize efficacy, prevent both relapse and undesired side-effects, ${ }^{9,14}$ and, consequently, improve treatment completion. A helpful tool to measure blood drug concentrations in the blood at a given time is the therapeutic drug monitoring (TDM). ${ }^{15}$ For instance, linezolid exhibits a high rate of myelotoxicity and peripherals neuropathy at high dosages while it displays a desirable blood concentration between 2 and $7 \mathrm{mg} / \mathrm{L}$ where the majority of AEs are avoided in bacterial infections other than TB. The use of linezolid coupled with the implementation of an adequate TDM sample timing immediately before the administration of the antibiotic may be helpful in adjusting its dosage, thus preventing side effects of AEs. ${ }^{16,17}$ Ideally, in the near future biologic samples, such as saliva, could be used to indirectly assess linezolid blood concentration to prevent preventable AEs. ${ }^{18}$ Rapid turnaround of TDM helps to assess in real-time efficacy and/or prevent toxicity, giving a precious aid to clinicians in the choice of anti-TB treatments. The drugs which can benefit from TDM are reported in Table 1. Of note, TDM samples should be obtained correctly to minimize the risk of false-results due to inadequate sampling (eg patient taking rifampicin the morning of programmed follow-up visits and wait over 2 hours before having the TDM blood sample). 
Table I Proposed Therapeutic Drug Monitoring in DR-TB Tailored Regimens

\begin{tabular}{|c|c|c|c|c|}
\hline Compounds & Dosage & Time for Sampling & $\begin{array}{l}\text { Desirable TDM } \\
\text { Level (mg/L) }\end{array}$ & Reason \\
\hline Isoniazid & $300 \mathrm{mg}$ die & $\begin{array}{l}2 \text { hours post dose on empty stomach (every follow-up visit or in case } \\
\text { of toxicity) }\end{array}$ & $3-6$ & $E / T$ \\
\hline Rifampicin & $600 \mathrm{mg}$ die & $\begin{array}{l}2 \text { hours post dose on empty stomach (every follow-up visit or in case } \\
\text { of toxicity) }\end{array}$ & $8-15$ & $\mathrm{E} / \mathrm{T}$ \\
\hline Pyrazinamide & $\begin{array}{l}25 \mathrm{mg} / \mathrm{Kg} \\
(\mathrm{max} 2500 \mathrm{mg}) \mathrm{die}\end{array}$ & $\begin{array}{l}2 \text { hours post dose on full or empty stomach (every follow-up visit or } \\
\text { in case of toxicity) }\end{array}$ & $20-60$ & $\mathrm{E} / \mathrm{T}$ \\
\hline Ethambutol & $\begin{array}{l}25 \mathrm{mg} / \mathrm{Kg} \\
(\max 1600 \mathrm{mg}) \mathrm{die}\end{array}$ & $\begin{array}{l}2 \text { and } 6 \text { hours post dose on full or empty stomach (every follow-up } \\
\text { visit or in case of toxicity) }\end{array}$ & $20-60$ & $\mathrm{E}$ \\
\hline Levofloxacin & $\max 1000 \mathrm{mg}$ die & $\begin{array}{l}2 \text { hours post dose on full or empty stomach (every follow-up visit or } \\
\text { in case of toxicity) }\end{array}$ & $8-12$ & $\mathrm{E}$ \\
\hline Moxifloxacin & $\max 800 \mathrm{mg}$ die & $\begin{array}{l}2 \text { hours post dose on full or empty stomach (every follow-up visit or } \\
\text { in case of toxicity) }\end{array}$ & $3-5$ & $\mathrm{E}$ \\
\hline Clofazimine & $\max 100 \mathrm{mg}$ die & $\begin{array}{l}2 \text { hours post dose on full or empty stomach (every follow-up visit or } \\
\text { in case of toxicity) }\end{array}$ & $0.5-4$ & $\mathrm{~T}$ \\
\hline Linezolid & $\max 1200 \mathrm{mg}$ die & immediately before the dose ( 28 days or in case of toxicity) & $2-7$ & $\mathrm{~T}$ \\
\hline $\begin{array}{l}\text { Meropenem } \\
\text { c.i. }\end{array}$ & $\max 6 \mathrm{~g}$ die & during administration (every week) & $8-12$ & $\mathrm{E}$ \\
\hline Bedaquiline & \multirow{4}{*}{\multicolumn{2}{|c|}{ To be implemented }} & & $\mathrm{E} / \mathrm{T}$ \\
\hline Delamanid & & & & $E / T$ \\
\hline Pretomanid & & & & $\mathrm{E} / \mathrm{T}$ \\
\hline PAS & & & & $E / T$ \\
\hline
\end{tabular}

Notes: Data from these studies. ${ }^{19-28}$

Abbreviations: c.i., continuous infusion; E, efficacy; PAS, para-aminosalicylic acid; T, prevent toxicity; TDM, therapeutic drug monitoring.

Moreover, in high-resource settings with, generally, low TB incidence a suitable approach to further enhance treatment completion by reducing AEs occurrence would consider patient's genotype analysis. For example, polymorphisms in N-acetyltransferase (NAT) gene is thought to predict the occurrence of isoniazid-induced AEs, with low NAT activity (free isoform) linked to increased risk of hepatocellular impairment, mainly in individuals whose origin are from West Asia, South Asia, and South America. ${ }^{29}$ Likewise, the polymorphism of leukotriene A4 hydrolase (LTA4H), a key enzyme in the eicosanoid pathway, may predict an effective response in individuals from Asia; in fact, LTA4H activity may increase inflammation, suggesting, then, an intensive use of steroids in TB meningitis. More studies are needed to confirm the role of LTA4H in modulating inflammation and TB response. ${ }^{30}$

The route of administration for anti-TB drugs - intravenous versus oral - depends mostly on disease's severity, localization, absorption, and available drugs. As highlighted by the $\mathrm{WHO}$, an all-oral 6-9-month regimen including $\mathrm{Bdq}, \mathrm{Pa}$, and linezolid (BPaL) may be prescribed in patients with pre-XDR-TB without any previous exposure $(<2$ weeks of treatment) to Bdq and linezolid and under operational research circumstances. ${ }^{8}$ Nevertheless, in some cases, gastrointestinal absorption may be reduced, or oral administration may not feasible; hence, injectable agents could be alternatively administered while waiting to resolve the underlying cause. ${ }^{31,32}$

Finally, clinicians should investigate if patients have had any form of allergy, hypersensitivity or intolerance to whatever previous medicine. This will enable to reduce any preventable severe $\mathrm{AE}$ when tailoring anti-TB regimen. ${ }^{33}$

\section{Pathogen-Related Variables}

From a pathogen point of view, recent scientific advances have been achieved to improve the management of DR- 
TB patients. Rapid molecular tools offer rapid resistance prediction compared to phenotypic DST. However, they allow prompt resistance prediction for several drugs and, by interrogating a limited part of genome, do not infer comprehensive drug susceptibility. Genome sequencing has indeed revolutionized and accelerated the progress toward tailored treatment for patients with DR-TB. By surveying the full "resistome" of Mycobacterium tuberculosis, the whole genome sequencing (WGS), offers the most comprehensive approach to determine resistance, predict susceptibility for few well-defined drug targets, and facilitate the timely design of individualized regimens. However, the reliance on culture as an input for sequencing makes WGS implementation technically challenging in some setting as well as time consuming as it requires other 2-3 weeks. Targeted Next Generation Sequencing (NGS) represents an attractive alternative to perform multiple single target assays directly from sputum, without waiting for colonies formation in culture media, and thus drastically reducing turnaround time to few days. Additional aspects such as phylogenetic lineage coupled with virulence and transmission properties are needed to move toward individualized regimen. In fact, although highly clonal, clinical Mycobacterium tuberculosis strains have significant genetic diversity resulting in relevant impact on virulence, transmission and mutation rate. $^{24,25}$ Despite NGS technologies are currently affordable, issues in the implementation (eg, infrastructure, human capacity, and data management) should be carefully considered. ${ }^{36}$

Therefore, together with DR profile, these additional aspects could significantly contribute to reducing treatment failure and build customized treatment.

\section{The Follow-Up}

Once the regimen for DR-TB has been tailored, efforts should be made to keep patients' adherent to it. If the patient has been hospitalized, he/she should be discharged continuing the treatment as out-patients to avoid any further costs for the hospital. ${ }^{34}$ Then, follow-up visits, including a clinical, radiological and microbiological reassessment, refill of the drugs used in his personalized antiTB regimen, TDM of the drugs, blood-test should be performed to adjust the duration of the treatment. To enhance monitoring, clinicians should be able to track patients' progress using electronic tools to increase adherence and reduce dropouts. ${ }^{37,38}$ Medical examination and biochemical and microbiological results from the follow- up visit can be made available to the patient through mobile text message in order to stimulate adherence to treatment and to follow-up. ${ }^{39}$

According to the localization of the disease, time to culture conversion, radiological improvement, clinical stability and good tolerance to treatment, it is possible to forecast a shortening in DR-TB treatment. ${ }^{40,41}$

\section{Challenges and Gaps in Providing Precision TB Treatment}

The idea behind standardization in TB and LTBI treatment is to adequately and easily provide a proven effective therapy to anyone without waiting for DST results and regardless of socio-cultural setting and available resources in the country where they operate.

Going back to a heterogeneous landscape of anti-TB regimens will require the draft and dissemination of solid and complex guidelines by policy makers and national public health authorities. However, such guidelines need to be coupled with training of the medical staff - both specialists and general practitioner - to adequately tailor the treatment.

Tailoring treatment to either host and pathogen biomarkers requires the availability of novel compounds and their wide production and distribution. In recent years, as previously mentioned, three novel compounds have been developed after more than 30 years with no progress in pharmaceutical field.

The novel anti-TB drugs have been licensed by the Regulators (Food and Drugs Administration and European Medicines Agency) without completing the whole R\&D process: Bdq was approved by FDA after a Phase 2 trial; Dlm was approved by EMA after a phase 2 trial; and, PA- 824 was approved by FDA after a phase $2 \mathrm{~b}$ trial. Consequently, marketing authorization alone fails in made new compounds, or regimens, widely available to patients and clinicians because policy makers, which use the GRADE (Grading of Recommendations, Assessment, Development and Evaluation) methodology, are often unable to timely assess the effectiveness of a novel regimen due to stringent criteria adopted in clinical trials. Therefore, to tailor anti-TB treatment a more agile and well-design R\&D mechanism is mandatory.

CRISPR-based technologies may be a promising tool to expand TB drug pipeline. CRISPR-MTB test is under development. However, shortcomings of the technology cannot 
allow its point-of-care implementation, integrated into a compact desktop machine for a sample-in-result-out assay. ${ }^{40}$

Following the path marked by the oncology to customize the treatment; TB care is now facing a revolution of thought. To date, there are an increasing number of clinical trials using Bayesian adaptative randomization to rapidly select promising compounds concurrently requiring a smaller sample size. Nevertheless, there is a limited experience in applying those stratification algorithms, thus reducing confounding variables, in anti-TB drug development. $^{41}$

From a pathogen's biomarkers viewpoint, to use a more sophisticated method to rapidly and accurately predict drug susceptibility, for example, by using WGS and NGS technologies in clinical practice as well in clinical trials, further items are needed. Firstly, the current nucleic acid amplification technologies (NAATs) (eg the cartridge Xpert MTB/RIF) due to their high sensitivity can detect nonviable bacilli leading to false-positive cases and unnecessary treatment, if used alone. Moreover, to use NAATs in drug R\&D process as a surrogate endpoint further research and validation are needed to replace the current standard based on culture. However, NAATs can be implemented to assess the emerge of new forms of drug-resistance, and the genotypic profile associated with it, when TB strains are exposed to a novel molecule during clinical trials thus concurrently developing a diagnostic tool to strengthen antimicrobial surveillance systems if the compounds achieve the market approval.

Furthermore, tailoring anti-TB drug based on NAAT results will require, if novel drugs will be available, an increased expertise by clinician or the development to assist them when fashioning the regimen.

Assessing host biomarkers, however, will remain challenging mostly because patient's willingness to disclose crucial information (eg alcohol intake) relies on the attitude of clinician. TDM can be expensive and not always feasible; especially at district level where transportation issues to reference laboratories can affect its utility.

A patient-centered approach will also increase adherence throughout the whole duration of the treatment and should also be considered during clinical trials. By doing that healthcare workers should be trained to adequately conduct the treatment prescription and monitoring. Nevertheless, follow-up visits should not be too frequent in case patients living far distant to clinics with a lack of public transports or social protection to afford the travel expenditures. Finally, special at-risk groups, such as homelessness, people living in overcrowded houses, and pregnant women should be actively screened for both LTBI and active TB to decrease the burden of disease.

\section{Conclusion}

Even if the complexity and the costs to develop novel compounds as well as the difficulties in create dedicated centers for DR-TB diagnosis and treatment worldwide may slow-down the path to tailored anti-TB treatment, to achieve the ambitious goal of TB elimination, a radical change in TB treatment, in order to give a more comprehensive approach based both on patients' peculiarities and driven by DST and WGS, is needed.

\section{Disclosure}

The authors report no conflicts of interest in this work.

\section{References}

1. World Health Organization. Documentation for World Health Assembly 67 (A67/11).Geneva: World Health Organization; 2014.

2. World Health Organization. Global tuberculosis report 2019. (WHO/ CDS/TB/2019.15).Geneva: World Health Organization; 2019.

3. Riccardi N, Alagna R, Saderi L, et al., for StopTB Italia Onlus Group. Towards tailored regimens in the treatment of drug-resistant tuberculosis: a retrospective study in two Italian reference Centres. BMC Infect Dis. 2019;19(1):564. doi:10.1186/s12879-019-4211-0

4. Villa S, Ferrarese M, Sotgiu G, et al. Latent tuberculosis infection treatment completion while shifting prescription from isoniazid-only to rifampicin-containing regimens: a two-decade experience in Milan, Italy. J Clin Med. 2020;9(1):101. doi:10.3390/jcm9010101

5. Villa S, Codecasa LR, Faccini M, et al. Tuberculosis among asylum seekers in Milan, Italy: epidemiological analysis and evaluation of interventions. Eur Respir J. 2019;54(4):100896. doi:10.1183/1399 3003.00896-2019

6. Skripconoka V, Danilovits M, Pehme L, et al. Delamanid improves outcomes and reduces mortality in multidrug-resistant tuberculosis. Eur Respir J. 2013;41(6):1393-1400. doi:10.1183/09031936.00125812,

7. Pinto L, Menzies D. Treatment of drug-resistant tuberculosis. Infect Drug Resist. 2011;4:129-135. doi:10.2147/IDR.S10332

8. WHO consolidated guidelines on tuberculosis. Module 4: Treatment Drug-Resistant Tuberculosis Treatment. Licence: CC BY-NC-SA 3.0 IGO. Geneva: World Health Organization;2020.

9. Wang MG, Huang WW, Wang Y, et al. Association between tobacco smoking and drug-resistant tuberculosis. Infect Drug Resist. 2018;11:873-887. doi:10.2147/IDR.S164596

10. World Health Organization. Treatment of Tuberculosis: guidelines 4th edition. (WHO/HTM/TB/2009.420). Geneva: World Health Organization; 2010.

11. Zumla A, Ippolito G, Ntoumi F, et al. Host-directed therapies and holistic care for tuberculosis. Lancet Respir Med. 2020;8(4):337-340. doi:10.1016/S2213-2600(20)30078-3

12. Alsultan A, Et Peloquin CA. Therapeutic drug monitoring in the treatment of tuberculosis: an update. Drugs. 2014;74(8):839-854. doi:10.1007/s40265-014-0222-8

13. Sotgiu G, Alffenaar JW, Centis R, et al. Therapeutic drug monitoring: how to improve drug dosage and patient safety in tuberculosis treatment. Int $J$ Infect Dis. 2015;32:101-104. doi:10.1016/j. ijid.2014.12.001 
14. Byng-Maddick R, Et Noursadeghi M. Does tuberculosis threaten our ageing populations? BMC Infect Dis. 2016;16(1):119. doi:10.1186/ s12879-016-1451-0

15. Alffenaar JC, Akkerman OW, Kim HY, Tiberi S, Migliori GB. Precision and personalized medicine and anti-TB treatment: is TDM feasible for programmatic use? Int J Infect Dis. 2020;92:S5S9. doi:10.1016/j.ijid.2020.01.041

16. Dryden MS. Linezolid pharmacokinetics and pharmacodynamics in clinical treatment. J Antimicrob Chemother. 2011;\%1 di \%266 Suppl 4:iv7-iv15. doi:10.1093/jac/dkr072

17. Pea F, Furlanut M, Cojutti P, et al. Therapeutic drug monitoring of linezolid: a retrospective monocentric analysis. Antimicrob Agents Chemother. 2010;54(11):4605-4610. doi:10.1128/AAC.00177-10

18. van den Elsen SHJ, Oostenbrink LM, Heysell SK, et al. Systematic review of salivary versus blood concentrations of antituberculosis drugs and their potential for salivary therapeutic drug monitoring. Ther Drug Monit. 2018;40(1):17-37. doi:10.1097/FTD.0000000000000462

19. Mota L, Al-Efraij K, Campbell JR, Cook VJ, Marra F, Johnston J. Therapeutic drug monitoring in anti-tuberculosis treatment: a systematic review and meta-analysis. Int J Tuberc Lung Dis. 2016;20 (6):819-826. doi:10.5588/ijtld. 15.0803

20. Peloquin CA. Therapeutic drug monitoring in the treatment of tuberculosis. Drugs. 2002;62(15):2169-2183. doi:10.2165/ 00003495-200262150-00001

21. Heysell SK, Moore JL, Staley D, Dodge D, Houpt ER. Early Therapeutic Drug Monitoring for Isoniazid and Rifampin among Diabetics with Newly Diagnosed Tuberculosis in Virginia, USA. Tuberc Res Treat. 2013;2013:129,723. doi:10.1155/2013/129723

22. Ebers A, Stroup S, Mpagama S, et al. Determination of plasma concentrations of levofloxacin by high-performance liquid chromatography for use at a multidrug-resistant tuberculosis hospital in Tanzania. PLoS One. 2017;12(1):e0170663. doi:10.1371/journal. pone. 0170663

23. Naidoo A, Naidoo K, McIlleron H, Essack S, Padayatchi N. A Review of Moxifloxacin for the Treatment of Drug-Susceptible Tuberculosis. J Clin Pharmacol. 2017;57(11):1369-1386. doi:10.1002/jcph.968

24. Davies Forsman L, Niward K, Hu Y, et al. Plasma concentrations of second-line antituberculosis drugs in relation to minimum inhibitory concentrations in multidrug-resistant tuberculosis patients in China: a study protocol of a prospective observational cohort study. BMJ Open. 2018;8(9):e023899. doi:10.1136/bmjopen-2018-023899

25. Pea F, Furlanut M, Cojutti $\mathrm{P}$, et al. Therapeutic drug monitoring of linezolid: a retrospective monocentric analysis. Antimicrob Agents Chemother. 2010;54(11):4605-4610. doi:10.1128/AAC.00177-10

26. Bolhuis MS, Tiberi S, Sotgiu G, et al. Linezolid tolerability in multidrug-resistant tuberculosis: a retrospective study. Eur Respir J. 2015;46(4):1205-1207. doi:10.1183/13993003.00606-2015

27. Schoenenberger-Arnaiz JA, Ahmad-Diaz F, Miralbes-Torner M, et al. Usefulness of therapeutic drug monitoring of piperacillin and meropenem in routine clinical practice: a prospective cohort study in critically ill patients. European Journal of Hospital Pharmacy. 2020;27:e30-e35.

Infection and Drug Resistance

\section{Publish your work in this journal}

Infection and Drug Resistance is an international, peer-reviewed openaccess journal that focuses on the optimal treatment of infection (bacterial, fungal and viral) and the development and institution of preventive strategies to minimize the development and spread of resistance. The journal is specifically concerned with the epidemiology of
28. Scharf C, Paal M, Schroeder I, et al. Therapeutic Drug Monitoring of Meropenem and Piperacillin in Critical Illness-Experience and Recommendations from One Year in Routine Clinical Practice. Antibiotics (Basel). 2020;9(3):131. doi:10.3390/antibiotics9030131

29. Zhang M, Wang S, Wilffert B, et al. The association between the NAT2 genetic polymorphisms and risk of DILI during anti-TB treatment: a systematic review and meta-analysis. Br J Clin Pharmacol. 2018;84(12):2747-2760. doi:10.1111/bcp.13722

30. Fava VM, Schurr E. Evaluating the impact of LTA4H genotype and immune status on survival from tuberculous meningitis. J Infect Dis. 2017;215(7):1011-1013. doi:10.1093/infdis/jix052

31. Goldani LZ, Spessatto CO, Nunes DL, et al. Management of severe gastrointestinal tuberculosis with injectable antituberculous drugs. Trop Med Health. 2015;43(3):191-194. doi:10.2149/ tmh.2015-09

32. Cherenko S, Lytvynenko N. Efficacy of intravenous administration of anti-tuberculosis drugs in patients with multidrug-resistant tuberculosis. Eur Respir J. 2013;42:P2814.

33. Chantaphakul H, Cheungpasitporn W, Ruxrungtham K, Klaewsongkram J. Adverse reaction to anti-tuberculosis drugs. J Allergy Clin Immunol. 2010;125(2):AB154. doi:10.1016/j.jaci.2009.12.604,

34. Cabibbe AM, Walker TM, Niemann S, Cirillo DM. Whole genome sequencing of Mycobacterium tuberculosis. Eur Respir J. 2018;52:1801163. doi:10.1183/13993003.01163-2018

35. de Steenwinkel JE, Ten Kate MT, de Knegt GJ, et al. Drug susceptibility of Mycobacterium tuberculosis Beijing genotype and association with MDR TB. Emerg Infect Dis. 2012;18:660-663. doi:10.3201/eid1804.110912

36. Riccardi N, Giannini B, Borghesi ML, et al. Time to change the single-centre approach to management of patients with tuberculosis: a novel network. ERJ Open Res. 2018;\%1 di \%24(1):00108-2017. doi:10.1183/23120541.00108-2017

37. Giannini B, Riccardi N, Cenderello G, Di Biagio A, Dentone C, Giacomini M. From liguria HIV web to liguria infectious diseases network: how a digital platform improved doctors' work and patients' care. AIDS Res Hum Retroviruses. 2018;34(3):239-240. doi:10.1089/aid.2017.0064

38. Elangovan R, Et Arulchelvan S. A study on the role of mobile phone communication in tuberculosis DOTS treatment. Indian J Community Med. 2013;38(4):229-233. doi:10.4103/0970-0218.120158

39. Riccardi N, Del Puente F, Magnè F, Taramasso L, Di Biagio A. Bedaquiline: a new hope for shorter and better anti-tuberculosis regimens. Recent Pat Antiinfect Drug Discov. 2018;13(1):3-11. doi:10.2174/1574891X12666170619101904

40. Rock J. Tuberculosis drug discovery in the CRISPR era. PLoS Pathog. 2019;15(9):e1007975. doi:10.1371/journal.ppat.1007975

41. Warner DF, Et Mizrahi V. Shortening treatment for tuberculosis back to basics. N Engl J Med. 2014;371(17):1642-1643. doi:10.1056/ NEJMe1410977 antibiotic resistance and the mechanisms of resistance development and diffusion in both hospitals and the community. The manuscript management system is completely online and includes a very quick and fair peerreview system, which is all easy to use. Visit http://www.dovepress.com/ testimonials.php to read real quotes from published authors. 